\title{
La educación superior: Liderazgo y competitividad en la educación financiera
}

\author{
Higher education: Leadership and competitiveness in financial education \\ Ensino superior: liderança e competitividade na educação financeira
}

María Emperatriz Fuertes Narváez ${ }^{1}$

mfuertes@liceoaduanero.edu.ec https://orcid.org/0000-0001-7967-9834

\author{
Brian Santiago Alarcón Gudiño ${ }^{1}$ \\ brian.alarcon@liceoaduanero.edu.ec \\ https://orcid.org/0000-0002-0221-5166
}

\section{Santiago Patricio López Chamorro² \\ splopez@utn.edu.ec \\ https://orcid.org/0000-0002-7772-3640}

\author{
${ }^{1}$ Instituto Superior Tecnológico Liceo Aduanero, Ibarra, Ecuador \\ ${ }^{2}$ Universidad Técnica del Norte, Ibarra, Ecuador
}

Artículo recibido en junio 2021, revisado en julio 2021, arbitrado en agosto 2021 y publicado en septiembre 2021

\section{RESUMEN}

La presente investigación tuvo por objetivo demostrar la incidencia del liderazgo y la competitividad en la educación financiera, como aporte de la aplicación de teorías y prácticas que permita alcanzar la proactividad, gestión $\mathrm{y}$ desarrollo en el Instituto Superior Tecnológico Liceo Aduanero. La metodología fue cualitativa, tipo descriptiva etnográfica. Los resultados se concentraron en competencias para mejorar el desempeño y el autoconocimiento en la toma de decisiones. Para cumplir con los objetivos, fue necesario que el líder se comprometiera con el aprendizaje continuo, la madurez afectiva y el desarrollo, como complementos. En conclusión, se estudió a la proactividad, gestión, desarrollo y acción personal como las competencias que determinan el nivel de liderazgo personal de un directivo. Contar con los elementos que conforman al líder proporciona óptimos resultados dentro de la institución; fue relevante pues el gerente es una figura capaz de auto conducirse para poder dirigir a los demás.

Palabras clave: Educación financiera; Gestión; Liderazgo; Cima organizacional; Administración en la organización
ABSTRACT

The objective of this research was to demonstrate the incidence of leadership and competitiveness in financial education, as a contribution to the application of theories and practices that allow achieving proactivity, management and development in the Instituto Superior Tecnológico Liceo Aduanero. The methodology was qualitative, ethnographic descriptive type. The results were focused on skills to improve performance and self-knowledge in decision-making. To meet the objectives, it was necessary for the leader to commit to continuous learning, affective maturity and development, as complements. In conclusion, proactivity, management, development and personal action were studied as the competencies that determine the level of personal leadership of a manager. Having the elements that make up the leader provides optimal results within the institution; it was relevant because the manager is a figure capable of conducting himself in order to direct others.

Key words: Financial education; management; leadership; organizational top; administration in the organization

\section{RESUMO}

O objetivo desta pesquisa foi demonstrar a incidência da liderança e da competitividade na educação financeira, como contribuição para a aplicação de teorias e práticas que permitem a proatividade, gestão e desenvolvimento no Instituto Superior Tecnológico Liceo Aduanero. A metodologia foi qualitativa, do tipo descritiva etnográfica. Os resultados foram focados em habilidades para melhorar o desempenho e o autoconhecimento na tomada de decisões. Para cumprir os objetivos, era necessário que o líder se comprometesse com o aprendizado contínuo, o amadurecimento afetivo e o desenvolvimento, como complementos. Em conclusão, proatividade, gestão, desenvolvimento e ação pessoal foram estudados como as competências que determinam o nível de liderança pessoal de um gestor. Ter os elementos que compõem o líder proporciona ótimos resultados dentro da instituição; Foi relevante porque o gerente é uma figura capaz de se conduzir para direcionar os outros.

Palavras-chave: Educação financeira; Gestão; Liderança; Topo organizacional; Administração na organização 


\section{INTRODUCCIÓN}

Uno de los principios de la administración en la organización depende de un líder que dinamice el trabajo en un ambiente motivado. El clima laboral aporta en el bienestar de las personas dentro de una organización orientado a hacer sentir al empleado cómodo y a gusto dentro de la misma. Una buena administración conlleva a un eficiente y estable ambiente laboral en todas las áreas y espacios de trabajo. La administración será efectiva cuando se cuente con personas líderes y la productividad será el fruto de una dirección focalizada. El trabajo productivo se nutre de un equipo de trabajo y un buen líder por lo cual es clave mencionar que el liderazgo se relaciona con el arte de influir en los individuos de tal forma que se sientan incentivados y motivados. Direccionar a los colaboradores a conocer lo que tienen que hacer y una vez que lo están realizando dejarles hacer interfiriendo lo menos posible y aceptando que se puedan equivocar.

Las organizaciones dependen en gran medida de su posibilidad de cambio y su capacidad financiera. Está capacidad de adaptación a las demandas del entorno y de su flexibilidad para afrontar la incertidumbre, depende de la forma en que las organizaciones son lideradas. Tradicionalmente las organizaciones son un modelo de cambio organizacional del rol del líder que adquiere. La organización enfrenta el cambio con la intención de controlarlo y devolver la estabilidad a la organización, mediante la orientación de acciones para el logro de unos resultados previamente establecidos (Contreras y Barbosa, 2013, p. 154). Las organizaciones permiten mantener satisfechos a sus clientes mediante amplios consensos respecto a la supervivencia y permanencia a sobrevivir en sus mercados adoptando novedosos y variados enfoques.
La gestión permite enfocarse en quienes planifican, organizan, dirigen, controlan y miden los resultados del talento humano. Esta identifica las características que se definen de acuerdo con el proceso de formación que han tenido dentro de su trayectoria profesional y experiencias de vida desde su niñez. Por lo tanto, permite generar una gran responsabilidad en el logro de objetivos que se identifican y se encuentran en la capacidad de dirigir equipos de trabajo. Una gestión correcta genera un enfoque de éxito que se basa inicialmente en conocer las habilidades de cada uno de sus coadjutores identificando las destrezas que los destacan y de igual forma en mantener un buen clima laboral. Esta actitud produce confianza en las personas, pues se toma conciencia sobre el logro de las metas cuando hay decisión y se está sujeto a grandes cambios humanísticos personales que se repliquen con las personas que lo rodean.

El liderazgo presenta estrategias aplicadas y enfocadas al crecimiento económico progresivo. También permite conocer la forma de representar a directivos y a equipos de personas. El mismo representa algunas reflexiones teóricas en direccionamiento de los colaboradores. La práctica de liderazgo enfoca escenarios que no todos están en condiciones de asumirlo entre ellos existen quienes el trabajo lo desempeñan bajo una orden. Esta acción se convierte en una herramienta fundamental para la gerencia del servicio por cuanto permite alinear al interés de los colaboradores. Dentro de los objetivos crean una visión y unas condiciones de trabajo atractivas generadoras de valor para el cliente. Además, identifica cómo el clima laboral influye en la productividad y en la contribución de ideas innovadoras con fines de crecimiento personal, profesional y empresarial. 
El clima organizacional de la educación superior y financiera refleja los valores las actitudes y las creencias de los miembros que se transforman a su vez en elementos del clima. Adicionalmente, prevé los problemas que puedan surgir frente a la organización esto permite sostener un cambio que indique al administrador los elementos específicos sobre los cuales debe dirigir sus intervenciones. Además, permite una planeación estratégica de la actividad. El desarrollo organizacional se basa en la hipótesis en la cual cada etapa adapta una forma organizacional que es la adecuada para esa fase en particular. Estos dependen de los cambios que se presentan y demandan revitalizar y construir nuevos modelos organizacionales. Por otra parte, requiere de una conciencia social del personal en las organizaciones, ya que es esencial en el mundo actual.

La educación financiera contribuye a reducir las barreras a la demanda de la inclusión financiera. (García et al., 2013), indican que en esta medida la educación financiera puede aumentar el conocimiento y la comprensión de los productos y servicios financieros promoviendo la demanda de estos. Frente a la protección del consumidor financiero, la educación financiera puede proporcionar a las personas el conocimiento de sus derechos y la comprensión de las obligaciones de las entidades financieras. El tema financiero constituye un complemento a la regulación de los mercados financieros y a las intervenciones públicas en este sector (p. 11). Todas las decisiones financieras implican un elemento financiero por desgracia, los estudios sobre cultura financiera muestran que incluso los conceptos financieros básicos no son claramente comprendidos por mucha gente. La ausencia de información hace que las personas sean vulnerables y en última instancia puede conducir a problemas de endeudamiento excesivo y exclusión financiera.
Las empresas creadas por emprendedores o directivos deben contar con líderes cuyo propósito sea conocer los movimientos económicos de la organización que dirige. Por lo que desde la posición de Ferrada (2018) aporta que la cultura en educación financiera es una competencia clave en la sociedad del siglo XXI, debido a la presencia permanente de las finanzas en la vida de cada persona, y la interacción que se establece en la adquisición de bienes y servicios, así como su relación con el bienestar personal. Adquirir conocimientos relacionados con la Educación Financiera favorece la realización de elecciones informadas (p. 61). La educación financiera no será eficiente por sí sola sino viene acompañada de una legislación o de entidades financieras que aporten para el desarrollo. Mejía (2016) afirma que "la transformación productiva ha sido una de las áreas que, como banco de desarrollo de América Latina, ha promovido como condición necesaria para alcanzar un desarrollo alto y sostenible" (p. 7).

Formarse en educación financiera constituye un valor agregado para el líder por lo tanto el liderazgo se concibe desde diferentes perspectivas. Las atribuciones generan desde un punto de vista formal y funcional confianza en el líder quien en su gestión tiene como responsabilidades la administración de los recursos, elaborar planes con relación a los objetivos. El ámbito educativo influye y no se deslinda de lo empresarial, las finanzas y la gestión con liderazgo y empoderamiento de empresas de producción, comercialización y servicios permitirá la generación de productividad (Amador, 2017, p. $3)$.

Dentro de la exposición problemática se tomó en cuenta que los equipos contemplan varias etapas en su desarrollo: pasan de la inmadurez a la madurez, guiados por un líder en el aprovechamiento de sus conocimientos y habilidades. Dichas etapas se explican a continuación: 


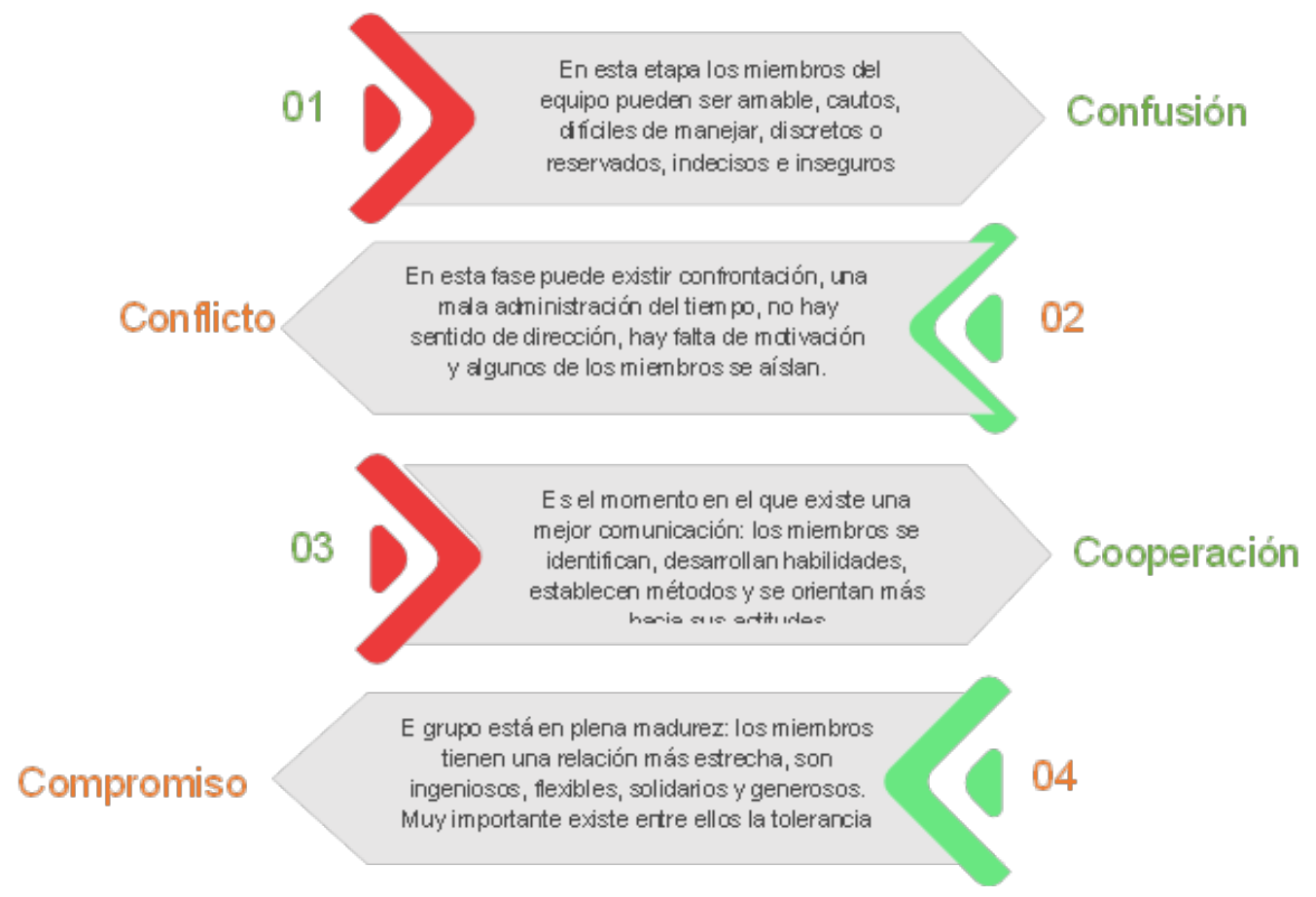

Figura 1. Fases y procesos en el desarrollo de un equipo. (Fuente: Villar, B. 2012. Fases y procesos en el desarrollo de un equipo).

Se debe explicar que de llegar a surgir un periodo de estancamiento, cuando existe un líder muy dominante que ejerce presión y control sobre el grupo y los miembros se limitan únicamente a obedecer las órdenes. Este estado puede darse en la etapa inicial de confusión, cuando los miembros son inseguros y fácilmente obedecen a la disposición de una persona. Cuando algunos integrantes transitan por este mismo estado, puede producirse consenso, porque en ese momento no existe un buen líder. Generalmente los equipos de trabajo llegan al conflicto, pero si existe una excelente coordinación, se puede evitar.

En este sentido, el objetivo de la presente investigación fue identificar la incidencia del liderazgo y la competitividad en la educación financiera, como aporte de la aplicación de teorías y prácticas que permita alcanzar la proactividad, gestión y desarrollo en el Instituto Superior Tecnológico Liceo Aduanero. La hipótesis que se planteó permite identificar de qué manera el liderazgo y la competitividad en la educación financiera impacta en el desarrollo organizacional del Instituto Superior Tecnológico Liceo Aduanero.

Es relevante el presente estudio porque sus hallazgos podrán ser utilizados en otros trabajos de investigación para dar soluciones argumentadas a fin de mejorar los procesos organizativos y de liderazgo en las instituciones de educación superior.

\section{MÉTODO}

La tipología que presenta este artículo permitió abordar los conceptos de la investigación cualitativa de corte descriptivo etnográfico, siendo el punto de partida que caracterizó el comportamiento del equipo de trabajo del Instituto Superior Tecnológico Liceo Aduanero durante el año 2020. Este proceso consideró extraer conclusiones y generalizaciones a partir de los datos estudiados fundamentando así el propósito de la investigación en cuanto a 
la incidencia en los procesos de gestión, clima organizacional, educación financiera y el andamiaje de actitudes y aptitudes de este grupo de estudio.

La estrategia principal para desarrollar esta investigación fue el uso del método comparativo constante, el cual generó en el investigador verificar, codificar y analizar los resultados para desarrollar conceptos, mediante la comparación de incidentes específicos de los datos, lo que posibilitó identificar y explorar sus interrelaciones en una teoría coherente, y la observación directa del comportamiento de cada uno de los miembros del equipo docente y administrativo bajo un análisis de comportamiento, trabajo en equipo, integración, desempeño profesional, actitud, compromiso, y el liderazgo dejando al lector que juzgue la credibilidad de los estudios cualitativos presentados en este artículo.

\section{RESULTADOS Y DISCUSIÓN}

Dentro de los resultados alcanzados desde la profesión se evidencia que el trabajo en equipo dentro de la institución de educación superior del Instituto Superior Tecnológico Liceo Aduanero, ha sido indispensable mejorar la experiencia de conocer a la organización, así como a personas de todos los géneros también fue fundamental para "abrir la mente" con el propósito de pensar en bienestar, tranquilidad, buen ambiente laboral, igualdad y tener la oportunidad de ser mentores para desarrollar personas. Conocer con quien se trabaja exigió realizar un análisis y reconocimiento de las personas quienes conforman el equipo de trabajo, descubrir las habilidades y destrezas de cada colaborador; de igual manera conocer los estados emocionales; descubrir la forma de ser y de comportarse condujo a planificar tomando en cuenta todos esos aspectos.

Otro hallazgo es que la experiencia en la contabilidad y las finanzas permitió relacionar la profesión con el liderazgo que se ha convertido en una de las estrategias que han conducido por buen camino la productividad y crecimiento corporativo de la institución.

Cabe destacar que se considera el sometimiento a una persona a una rutina diaria sería como un castigo a una acción mal ejecutada. La educación financiera concientiza sobre lo que la persona es capaz como líder y profesional para desarrollarse y tener convicción de lograr hacer líderes a quienes trabajan en la institución. Es también relevante hacer ver a los colaboradores el límite entre la amistad y el trabajo. En vista de que un trabajador realiza mejor sus actividades cuando se encuentra en un buen ambiente de trabajo. Este aspecto se logró mediante la programación conjunta de actividades de esparcimiento, las cuales hicieron que todo el personal sienta pasión por lo que hace y espera dentro del mes. Fue un incentivo de compartir con los demás compañeros juegos, comidas, y actividades de recreación.

\section{Discusión}

En este artículo desde una perspectiva cualitativa se debate sobre la interpretación de la gerencia del servicio que le asigna al liderazgo de la educación superior financiera un rol más elevado, significativo y de mayor alcance. Discutir sobre lo que implica abandonar paradigmas tradicionales en la atención al cliente, para acercarse a un pensamiento innovador y cambio trascendente acorde a las nuevas exigencias del mercado. El liderazgo ocurre cuando un miembro de un grupo modifica la motivación o las competencias de otros en el grupo. Así pues, se observa, que el liderazgo involucra el uso de la influencia y que todas las relaciones interpersonales pueden involucrar el liderazgo. Igualmente, esta definición implica la relevancia de ser un agente de cambio, 
capaz de afectar el desempeño y la conducta de los seguidores. Uno de los objetivos de los directivos es contar con personas idóneas para cada puesto y tener en el equipo talentos con conocimientos financieros que les permita llevar paralelamente el ejercicio que permita incrementar la productividad y las finanzas. Para un líder ofrecer un servicio de excelente calidad se ha convertido en una estrategia de competitividad para las organizaciones de servicio.

Se coincide con Perdomo y Prieto (2009) porque se estudia los cambios que ha sufrido el consumidor, es más exigente en el momento de la compra, desea obtener mayores beneficios por la inversión a realizar. Al mismo tiempo, la competencia es más intensa, más agresiva ampliando las opciones para los clientes haciéndolos menos leales o conformistas.

En concordancia al contexto es importante también debatir en la educación financiera acerca de la pasión por el cliente; es necesario tener una clara orientación al servicio, desarrollar actitudes que favorezcan su posición sobre la acción de satisfacer las necesidades de otros, y moldearlas para lograr así un cambio cultural dentro de la organización basada en principios éticos que evidencien su integridad como persona y profesional. Creer en otros: los líderes orientados al servicio confían en las condiciones intrínsecas de las personas para llegar a una alta autorrealización, quienes tienen suficientes conocimientos y aptitudes para desarrollar e implantar una alta motivación al logro, conducente a la obtención de resultados de excelencia. Con estas competencias las personas están en capacidad de establecer un estilo de liderazgo participativo orientado a la excelencia de servicio, y con la creencia de que la gente es digna de confianza. De esta forma, se hace necesario superar las limitaciones propias de la complejidad de la investigación sobre educación financiera para:

\section{Establecer un proceso de comunicación con}

las siguientes características: 1) Contar con una finalidad claramente establecida 2) ser multidireccional, hacia todos los sentidos y niveles de la organización; 3) estar debidamente instrumentada, valiéndosedesoportes, herramientas y equipos, así como de indicadores seleccionados en función de los objetivos; 4) adaptada a los sistemas de información de la organización y 5) debe ser lo suficientemente flexible para integrar lo informal para crear estructuras que contribuyan a la consecución de la excelencia de servicio.

Querer el trabajo: los líderes exitosos orientados al servicio hacen lo que quieren, porque quieren lo que hacen, como tienen una alta motivación al logro se imponen riesgos de muchas complejidades, desarrollan una gran capacidad de prospección $\mathrm{y}$ asertividad $\mathrm{y}$, las incertidumbres las enfrentan con un gran deseo de triunfar y en verdad son triunfadores. El líder no sólo debe enseñar el funcionamiento del negocio, sino con el ejemplo debe mostrar su estilo, valores y excelencia.

Integridad: Es un concepto propio de líderes tienen grandes principios éticos y morales, en consecuencia, siempre están haciendo lo correcto y actuando de buena fe, concediéndole gran importancia al principio de justicia y equidad, así como también la coherencia y veracidad hacia los clientes, tanto internos como externos. La integridad como persona y como profesional es una cualidad esencial del liderazgo de servicio (Perdomo y Prieto, 2009).

Paralelamente a lo estipulado por Alanis et al., (2017) se comprendió como la evidencia del trabajo para compartir desde la acción de liderar a más de sesenta trabajadores donde todos reciben un trato con respeto y consideración; el personal se sintió atraído por el trabajo y, en consecuencia, lo desarrollan con gratitud hacia la institución. 
Actualmente, en las organizaciones y núcleos de trabajo, el liderazgo toma un papel fundamental. Se pasa de grupos de trabajo muy marcados por el descontento a grupos laborales que también necesitan sentirse queridos y bien tratados para rendir de una manera adecuada y satisfactoria, de cara a la consecución de los objetivos. La existencia de la figura de un líder puede repercutir de manera positiva en las organizaciones, ya que otorga un plus a los equipos que se sienten respaldados y bien entendidos por una figura superior en rango, que se preocupa por el bienestar de los empleados. Sin embargo, no siempre sucede esto, ya que algunas organizaciones se encuentran en muchas ocasiones dirigidas por líderes que carecen de la capacidad suficiente para gestionar los grupos de trabajo. El estudio planteado cobra relevancia sobre todo en los tiempos actuales.

Existe un punto de encuentro entre la presente investigación y lo argumentado por Jiménez y Villanueva (2018) puesto que se asume en el estudio de qué manera la evolución constante que están tomando las organizaciones, llevan a un aumento de la necesidad en la plantilla de la figura de un líder que sea capaz de gestionar al grupo. Un líder debe encontrar la manera más fácil de lograr los objetivos. Los estilos de liderazgo que se recogen en este trabajo son algunos de los estilos más representados en las distintas organizaciones. Cada estilo cuenta con unos condicionantes propios, con ventajas e inconvenientes en cada uno de ellos

De la misma forma en el marco del análisis de la educación financiera el liderazgo conlleva a la innovación por lo cual, "es indiscutible la necesidad del trabajo en equipo para cualquier actividad que se desempeñe, ya que los individuos en grupo tienen la capacidad para producir más y de manera más vertiginosa" en este sentido las empresas van a ser más competitivas y generaran mayor productividad contribuyendo al desarrollo socio económico del país. (Alanís et al., 2017, p. 406).

También fue beneficioso en el estudio tomar en cuenta los procesos de coaching para las organizaciones, debido a lo siguiente: A nivel organizativo facilita la transferencia del conocimiento, la colaboración y el acercamiento del personal en plantilla, así como el intercambio de experiencias; Promueve conversaciones orientadas hacia la consecución de objetivos corporativos, lo que genera grandes soluciones basadas en la creatividad y consolida el compromiso, espíritu de equipo y sentimiento de pertenencia; promueve el desarrollo de nuevas habilidades en la organización, lo que se traduce en mayores niveles de innovación y competitividad, y fomenta la cultura corporativa orientada al futuro, a la acción y a los resultados (Asociación Española de Coaching, 2018, p. 37). Al respecto la motivación hacia los equipos de trabajo orienta al logro de objetivos, un trabajador que se sienta importante dentro de la organización donde trabaja, desarrollará el máximo de su potencial físico e intelectual entregándolo en acciones para quién trabaja.

Finalmente coincidiendo con Torres et al., (2012) se caracteriza a los roles de liderazgo y género donde se toma en cuenta a las condiciones socioeconómicas y políticas en las que se encuentran inmersas las empresas hoy se caracterizan por un alto grado de incertidumbre, escaso nivel de predicción, entornos altamente complejos, globalización, economías emergentes e interdependientes, mayor competencia mundial, tendencias más globales que locales y cambios demográficos y tecnológicos. Estas circunstancias imponen a las organizaciones y a los líderes grandes e importantes retos. 
A continuación, en el marco de la discusión con los referentes de la investigación es importante destacar que hoy en día, los equipos de trabajo se han vuelto trascendentales en las organizaciones, constituyen los medios para lograr objetivos de mayor relevancia. Por esta razón se debe fomentar en la educación financiera las siguientes discusiones: Un equipo de trabajo es un grupo en el que los esfuerzos individuales dan como resultado un desempeño mayor que la suma de las aportaciones por individuo; un equipo es la unidad formada por dos o más personas con habilidades complementarias, que se comprometen en un propósito común y fijan objetivos y expectativas de desempeño comunes, de los cuales se responsabilizan; un equipo es un número reducido de personas con habilidades complementarias, comprometidas con un propósito común, una serie de metas de desempeño y un método de trabajo, del cual todas ellas son mutuamente responsables, y los equipos de trabajo en definitiva son la médula de la organización, cada integrante sino cuenta con habilidades las descubre si se da la oportunidad de dejar el miedo a un lado, ninguno nace con el conocimiento, la clave del éxito es demostrar valores, principios y cualidades en la ejecución de una acción.

Convertirse en un líder efectivo requiere de actitudes positivas hacia las demás personas que integran el equipo. Es igualmente importante fomentar el diálogo, observar lo que hacen las demás personas, autocriticarse y convertir obstáculos en oportunidades. Un grupo de trabajo en donde no exista un liderazgo efectivo es un equipo sin rumbo y sin dirección.

Siguiendo a Alhogbi (2017) los nuevos modelos de liderazgo en contraposición a los modelos tradicionales de liderazgo en los que las conductas de los líderes se basan en los intercambios que establecen con sus seguidores (en términos de intercambio de costes y beneficios, siguiendo el modelo de liderazgo transaccional). El lado humano de un líder muestra principios y formación.

Así, relacionar dos conceptos de liderazgo y educación financiera resulta llamativo porque en la práctica no son solo conceptos; se aplican a diario un líder debe tener conocimientos sólidos de los movimientos económicos que son parte del direccionamiento y responsabilidad de quien está a cargo de la dirección. Ejemplo un gerente, un rector, un director toma decisiones de acuerdo con los resultados económicos, es por ello que es recomendable que un líder tenga este tipo de instrucción para que la decisión que toma no la haga porque le sugieren los expertos sino porque conoce y tiene la certeza de un documento elaborado mas no maquillado.

De allí que, surge una nueva filosofía de gestión en las organizaciones de servicio denominada gerencia del servicio, la cual comienza con la naturaleza de la experiencia del cliente y crea estrategias y tácticas que maximizan la calidad de esa experiencia, optimizando la gestión de relaciones con los clientes, procurando su fidelización. En este sentido, se concibe la gerencia del servicio como un proceso integral que requiere de un decidido liderazgo por parte de los directivos de la empresa para que sus decisiones y acciones contribuyan a que los empleados sean más efectivos en los momentos de verdad. El liderazgo se convierte en una herramienta fundamental para la gerencia del servicio, por cuanto permite alinear el interés de los colaboradores con los objetivos de la organización creando una visión y unas condiciones de trabajo atractivas generador de valor para el cliente. 


\section{CONCLUSIONES}

Realizar esta investigación argumentativa sobre liderazgo, educación financiera y trabajo en equipo, permitió analizar temáticas referentes al trabajo directivo y colaborativo que se necesita para ejecutar objetivos de organizaciones, adaptado a estrategias financieras, estilos y enfoques aplicables en ejercicios reales y prácticos de las figuras de coordinadores, directores y subdirectores de instituciones académicas. El aporte teórico nutre a los gerentes y rectores; les otorga la capacidad para autoliderarse y mejorar su desempeño en la acción de liderar equipos de personas conformados por géneros distintos siendo útiles para ejecutar un trabajo equitativo. En una atmósfera razonable que compromete al líder, puesto que debe desarrollarse día a día con la contribución de ideas de todos los colaboradores. En el desarrollo de proyectos es cuando se conoce a las personas, así como también en el paso del tiempo. Algunos demuestran trabajo duro por unos meses por agradar a su superior y cuando encuentran estabilidad se direccionan al descuido y a la conformidad.

Contrastar los conceptos teóricos ha permitido conocer ideas y emitir críticas mediante análisis y aportes basados en la experiencia; además permitió comprender que un líder debe conocer a las personas y conocerlas para poder emprender liderazgo con ellos. De la misma forma en la constitución de equipos de trabajo para que ejecuten una tarea conjunta se considera necesario: la autonomía, para que el equipo tenga claro sus roles y funciones, capacidad para comunicarse e informar, la motivación que le permita enfrentar los cambios. También se concluye que los equipos de trabajo tienen compromisos compartidos entre sí para el desempeño colectivo, sin embargo, las metas no se alcanzan sin la interacción de los que forman el equipo.
La sinergia constituye un factor necesario entre todos los miembros para el logro de objetivos. De igual manera se concluye que los líderes están encaminados a llevar un equipo a la cima, a motivar, darles a conocer ideas, objetivos, metas y proyectos para que se empoderen y contribuyan con trabajo y descubran el líder que lleva cada uno adentro. En el análisis de los conceptos sobre liderazgo se estudia que un líder contagia de energía a sus colaboradores y busca desarrollar líderes que trabajen con actitud y pasión en cada puesto de trabajo. De igual manera se analizó que las teorías fundamentan las experiencias que se viven haciendo liderazgo, la investigación permitió identificar que un directivo es la persona que guía con métodos y herramientas a un equipo organizado, motivado y con conocimientos que aportan en creación, innovación, diseño e ideas que contribuyan al crecimiento corporativo $y$ productivo de la organización.

\section{REFERENCIAS}

Alanis, T., Idalia, N., y Luis, J. (2017). Trabajo en Equipo, Grupos de Trabajo y Perspectiva de Competencia. Daena: International Journal of Good Conscience, 12(3), 405-422. http://www. spentamexico.org/v12-n3/A25.12(3)405-422. pdf

Alhogbi, B. G. (2017). a. La Inclusión y calidad, la experiencia de las nuevas universidades en América Latina. En Journal of Chemical Information and Modeling (Vol. 53, Número 9). http://www.elsevier.com/locate/scp

Amador, O. C. M. (2017). Ventajas del liderazgo distribuido en instituciones de educación superior / Advantages of distributed leadership in institutions of higher education. RIDE Revista Iberoamericana para la Investigación y el Desarrollo Educativo, 8(15), 817-832. https:// doi.org/10.23913/ride.v8i15.322 
Asociación Española de Coaching. (2018). El libro blanco del coaching. En Asociación Española de Coaching, ASESCO. www.editorialcirculorojo. com

Contreras, T. F., y Barbosa, R. D. (2013). Del liderazgo transaccional al liderazgo transformacional: implicaciones para el cambio organizacional From Transactional Leadership to Transformational Leadership: Implications for Organizational Change $\mathrm{Du}$ leadership transactionnel au leadership transf. Revista Virtual Universidad Católica del Norte, 152-165. http://eds.a.ebscohost.com/ehost/pdfviewer/ pdfviewer?vid=21\&sid=8e66c179-034c-471aa 867 -da6da4e 1670 f\%40sessionmgr $4002 \&$ h $\mathrm{id}=4202$

Ferrada, C., y Díaz-levicoy, D. (2018). Chilenos de educación primaria Analysis of activities on financial education in chilean primary education textbooks. 65, 48-65

García, N., Grifoni, A., y López, J. (2013). La educación financiera en América Latina y el Caribe. Situación actual y perspectivas. En Banco de Desarrollo de América Latina (Vol. 12). http://www.oecd.org/daf/fin/financialeducation/OECD_CAF_Financial_Education_ Latin_AmericaES.pdf
Jiménez, A., y Villanueva, M. (2018). Los estilos de liderazgo y su influencia en la organización: Estudio de casos en el Campo de Gibraltar. Gestión Joven Revista de la Agrupación Joven Iberoamericana de Contabilidad $y$ Administración de Empresas, 18, 183-195. http:// elcriterio.com/revista/contenidos_18/13.pdf

Mejía, D. M., y Rodríguez, G. (2016). Determinantes socioeconómicos de la educación financiera

Perdomo, Y., y Prieto, R. (2009). El liderazgo como herramienta de competitividad para la gerencia del servicio. CICAG: Revista del Centro de Investigación de Ciencias Administrativas y Gerenciales, 6(2), 30-48

Torres, C., Ortiz, P., Eduardo, J., y Restrepo, M. (2012). La mujer y el liderazgo empresarial * Women and Business Leadership Resumen. 8, 183-194.

Villar, B. C. de J. (2012). Liderazgo Empresarial Cleopatra. En Red Tercer Milenio. http://www. aliat.org.mx/BibliotecasDigitales/economico_ administrativo/Liderazgo_empresarial.pdf 